\title{
Gromov-Witten theory of K3 surfaces and a Kaneko-Zagier equation for Jacobi forms
}

\author{
Jan-Willem van Ittersum ${ }^{1,2} \cdot$ Georg Oberdieck $^{3}$ - Aaron Pixton ${ }^{4}$
}

Accepted: 30 May 2021 / Published online: 2 July 2021

(C) The Author(s) 2021

\begin{abstract}
We prove the existence of quasi-Jacobi form solutions for an analogue of the KanekoZagier differential equation for Jacobi forms. The transformation properties of the solutions under the Jacobi group are derived. A special feature of the solutions is the polynomial dependence of the index parameter. The results yield an explicit conjectural description for all double ramification cycle integrals in the Gromov-Witten theory of K3 surfaces.
\end{abstract}

Mathematics Subject Classification $14 \mathrm{~J} 28 \cdot 14 \mathrm{~N} 35 \cdot 11 \mathrm{~F} 50 \cdot 11 \mathrm{~F} 11$

\section{Introduction}

\section{$1.1 \mathrm{~K} 3$ surfaces}

The Yau-Zaslow formula (proven by Beauville [2] and Bryan-Leung [3]) evaluates the generating series of counts of rational curves on $\mathrm{K} 3$ surfaces in primitive classes as the inverse of the discriminant

$$
\Delta(\tau)=q \prod_{n \geq 1}\left(1-q^{n}\right)^{24}
$$

$凶$ Georg Oberdieck

georgo@math.uni-bonn.de

Jan-Willem van Ittersum

j.w.m.vanittersum@uu.nl

Aaron Pixton

pixton@umich.edu

1 Mathematisch Instituut, Universiteit Utrecht, Postbus 80.010, 3508 TA Utrecht, The Netherlands

2 Max-Planck-Institut für Mathematik, Vivatsgasse 7, 53111 Bonn, Germany

3 Mathematisches Institut, Universität Bonn, Endenicher Allee 60, 53115 Bonn, Germany

4 Department of Mathematics, University of Michigan, Ann Arbor, USA

Birkhäuser 
where $q=e^{2 \pi i \tau}$ and $\tau \in \mathbb{H}$ is the standard variable of the upper half-plane.

More general curve counts on K3 surfaces are defined by the Gromov-Witten invariants

$$
\left\langle\alpha ; \gamma_{1}, \ldots, \gamma_{n}\right\rangle_{g, \beta}^{S}:=\int_{\left[\bar{M}_{g, n}(S, \beta)\right]^{\mathrm{red}}} \pi^{*}(\alpha) \prod_{i=1}^{n} \operatorname{ev}_{i}^{*}\left(\gamma_{i}\right)
$$

where $\bar{M}_{g, n}(S, \beta)$ is the moduli space of $n$-marked genus $g$ stable maps to a K3 surface $S$ representing the class $\beta \in H^{2}(S, \mathbb{Z})$, and

$$
\pi: \bar{M}_{g, n}(S, \beta) \rightarrow \bar{M}_{g, n}, \quad \mathrm{ev}_{i}: \bar{M}_{g, n}(S, \beta) \rightarrow S, i=1, \ldots, n
$$

are the forgetful and evaluation maps. The integral is taken over the reduced virtual fundamental class and the insertions are arbitrary classes

$$
\alpha \in H^{*}\left(\bar{M}_{g, n}\right), \quad \gamma_{1}, \ldots, \gamma_{n} \in H^{*}(S)
$$

Let $\mathrm{a}=\left(\mathrm{a}_{1}, \ldots, \mathrm{a}_{n}\right)$ be a list of integers with $\sum_{i} \mathrm{a}_{i}=0$. The moduli space $\bar{M}_{g, n}\left(\mathbb{P}^{1}, \mathrm{a}\right)$, defined in relative Gromov-Witten theory, parametrizes stable maps from a curve of genus $g$ to $\mathbb{P}^{1}$ with ramification profiles over 0 and $\infty$ given respectively by the positive and negative entries in a. The double ramification cycle

$$
\mathrm{DR}_{g}(\mathrm{a}) \in H^{2 g}\left(\bar{M}_{g, n}\right)
$$

is defined as the pushforward under the forgetful map $\bar{M}_{g, n}\left(\mathbb{P}^{1}, \mathrm{a}\right) \sim \bar{M}_{g, n}$ of the virtual class on this moduli space (see [6]).

Let also $z \in \mathbb{C}$ and $p=e^{z}$, and consider the odd (renormalized) Jacobi theta function

$$
\Theta(z, \tau)=\left(p^{1 / 2}-p^{-1 / 2}\right) \prod_{m \geq 1} \frac{\left(1-p q^{m}\right)\left(1-p^{-1} q^{m}\right)}{\left(1-q^{m}\right)^{2}} .
$$

The following formula was found in the study of the quantum cohomology of the Hilbert scheme of points of a K3 surface in [15], and related to K3 surfaces in [16].

Conjecture 1.1 ([15,16]) There exist quasi-Jacobiforms $\varphi_{m}(z, \tau), \varphi_{m, n}(z, \tau)$ such that for all primitive effective $\beta \in H^{2}(S, \mathbb{Z})$ we have

$$
\begin{aligned}
& \sum_{g=0}^{\infty}\left\langle\mathrm{DR}_{g}(\mathrm{a}) ; \gamma_{1}, \ldots, \gamma_{n}\right\rangle_{g, \beta}^{S}(-1)^{g+n} z^{2 g-2+n}=\frac{1}{\prod_{i} \mathrm{a}_{i}^{\operatorname{deg}\left(\gamma_{i}\right)}} \\
& \operatorname{Coeff}_{q^{\frac{1}{2} \beta^{2}}}\left(\sum_{\left\{\left(a_{j}, b_{j}\right)\right\}_{j},\left\{c_{j}\right\}_{j}} \frac{1}{\Theta^{2} \Delta} \prod_{j}\left(\gamma_{a_{j}}, \gamma_{b_{j}}\right) \varphi_{a_{j}, b_{j}} \cdot \prod_{j}\left(\gamma_{c_{j}}, \beta\right) \varphi_{c_{j}}\right) .
\end{aligned}
$$


Here, the sum on the right side is over all partitions of the set $\left\{\left(a_{i}, \gamma_{i}\right)\right\}_{i=1}^{n}$ into parts of size $\leq 2$. The parts of size 1 are labeled by $\left(c_{j}, \gamma_{c_{j}}\right)$, and the parts of size 2 are labeled $\left\{\left(a_{j}, \gamma_{a_{j}}\right),\left(b_{j}, \gamma_{b_{j}}\right)\right\}$. Moreover, $\operatorname{deg}(\gamma)$ denotes half the cohomological degree of $\gamma$, i.e. $\gamma \in H^{2 \operatorname{deg}(\gamma)}(S)$, and $(-,-)$ is the Mukai pairing on $H^{*}(S)$ defined by

$$
\left(\left(r_{1}, D_{1}, n_{1}\right),\left(r_{2}, D_{2}, n_{2}\right)\right)=r_{1} n_{2}+r_{2} n_{1}-D_{1} \cdot D_{2}
$$

where we write $D_{1} \cdot D_{2}=\int_{S} D_{1} \cup D_{2}$ for the intersection of divisors.

We refer to Sect. 2.3 for the definition of quasi-Jacobi forms. The left hand side of the conjecture is a (virtual) count of curves on K3 surfaces, whose normalization admits a map to $\mathbb{P}^{1}$ with prescribed ramification over two points of the target and with the ramification points incident to given cycles $\gamma_{i}$. If there are no marked points, the double ramification cycle is the top Chern class $\lambda_{g}$ of the Hodge bundle over the moduli spae of curves,

$$
\mathrm{DR}_{g}(\emptyset)=(-1)^{g} \lambda_{g}
$$

In this case the conjecture specializes to the Katz-Klemm-Vafa formula

$$
\sum_{g=0}^{\infty}\left\langle\lambda_{g}\right\rangle_{g, \beta}^{S} z^{2 g-2}=\text { Coeff }{ }_{q^{\frac{1}{2} \beta^{2}}}\left(\frac{1}{\Theta(z, \tau)^{2} \Delta(\tau)}\right) .
$$

proven in [12].

While the functions $\varphi_{m}, \varphi_{m, n}$ were conjectured to be quasi-Jacobi forms (of explicit weight and index) they have been left indeterminate in $[15,16]$. The goal of this paper is simply to give an explicit formula for these functions and study their properties.

\subsection{A Kaneko-Zagier equation for Jacobi forms}

Let $D_{\tau}=\frac{1}{2 \pi i} \frac{d}{d \tau}=q \frac{d}{d q}$ and consider the ratio

$$
F(z):=\frac{D_{\tau}^{2} \Theta(z)}{\Theta(z)}=-\sum_{n \geq 1} \sum_{d \mid n}(n / d)^{3}\left(p^{d / 2}-p^{-d / 2}\right)^{2} q^{n}
$$

where, as we will often do, have dropped $\tau$ from the argument.

We define formal series $\varphi_{m} \in \mathbb{Q}\left[p^{ \pm \frac{1}{2}}\right][[q]]$ for all $m \in \mathbb{Z}$ by the differential equation

$$
D_{\tau}^{2} \varphi_{m}=m^{2} F \varphi_{m}
$$

together with the constant term

$$
\varphi_{m}=\left(p^{m / 2}-p^{-m / 2}\right)+O(q) .
$$


Since the constant term of $F$ in $q$ vanishes, (1) determines the functions $\varphi_{m}$ uniquely from the initial data. By definition, we have $\varphi_{-m}=-\varphi_{m}$.

Our first main result is the following characterization of the functions $\varphi_{m}$.

Theorem 1.2 For all $m \geq 0$ we have

$$
\varphi_{m}=\operatorname{Res}_{x=0}\left(\frac{\Theta(x+z)}{\Theta(x)}\right)^{m} .
$$

In particular, $\varphi_{m}$ is a quasi-Jacobi form of weight -1 and index $|m| / 2$ for every $m$.

Consider the ratio of theta functions

$$
f(x)=\frac{\Theta(x+z)}{\Theta(x)}
$$

whose appearance in mathematics goes back to work of Eisenstein [19]. Since its inverse has Taylor expansion $1 / f(x)=\Theta(z)^{-1} x+O\left(x^{2}\right)$, the function $1 / f(x)$ can be formally inverted. By Lagrange inversion, Theorem 1.2 then precisely says that the inverse series is the generating series of the $\varphi_{m}$ :

$$
y=\frac{1}{f(x)} \Longleftrightarrow x=\sum_{m=1}^{\infty} \frac{\varphi_{m}}{m} y^{m} .
$$

Let us explain the connection of the differential equation (1) to a well-known differential equation for modular forms. Recall the Eisenstein series

$$
E_{k}(\tau)=1-\frac{2 k}{B_{k}} \sum_{n \geq 1} \sum_{d \mid n} d^{k-1} q^{n}
$$

where the weight $k \geq 2$ is even and $B_{k}$ are the Bernoulli numbers. Let

$$
\vartheta_{k}=D_{\tau}-\frac{k}{12} E_{2}(\tau)
$$

be the Serre derivative which restricts to an operator $\operatorname{Mod}_{k} \rightarrow \operatorname{Mod}_{k+2}$ on the space of modular forms of weight $k$. The Kaneko-Zagier equation [9] is the differential equation

$$
\vartheta_{k+2} \vartheta_{k} f_{k}=\frac{k(k+2)}{144} E_{4}(\tau) f_{k}
$$

If $k \equiv 0$ or $4 \bmod 6$ it has non-trivial solutions which are modular forms of weight $k$. A direct calculation shows that a function $f_{k}$ is a solution to (4) if and only if $g_{k+1}=$ $f_{k} / \eta^{2 k+2}$, with $\eta(\tau)=q^{1 / 24} \prod_{n \geq 1}\left(1-q^{n}\right)$ the Dedekind function, is a solution of

$$
D_{\tau}^{2} g_{m}=m^{2} \frac{E_{4}(\tau)}{144} g_{m} .
$$


We observe that the differential equation (1) is a Jacobi-form analogue of the Kaneko-Zagier equation. Even stronger, since (1) does not involve derivatives in the elliptic variable, we can specialise it to $\frac{z}{2 \pi i}=a$ for any $a \in \mathbb{Q}$ and in this way obtain an infinite family of Kaneko-Zagier type differential equations with modular solutions for a congruence subgroup. ${ }^{1}$ The inversion formula (3) has the classical analogue [9, Thm. 5(iv)]

$$
x=\sum_{k \geq 1} \frac{f_{k-1}}{k} y^{k} \Longleftrightarrow y=\left(\frac{\wp^{\prime}(x)}{-2}\right)^{-1 / 3}
$$

where the role of $f(x)$ is played by the formal cube root of the derivative $\wp^{\prime}(x)=$ $\frac{d}{d x} \wp(x)$ of the Weierstrass elliptic function $\wp(x)$, and the solutions $f_{k}$ are normalized accordingly.

We refer to Sect. 5 for a general construction of differential equations of KanekoZagier type.

\subsection{Differential equation of the second kind}

We are also interested in a second family of functions, defined in terms of the $\varphi_{m}$ of the previous section.

Define formal series $\varphi_{m, n} \in \mathbb{Q}\left[p^{ \pm 1 / 2}\right][[q]]$ for all $m, n \in \mathbb{Z}$ by the differential equation

$$
D_{\tau} \varphi_{m, n}=m n \varphi_{m} \varphi_{n} F+\left(D_{\tau} \varphi_{m}\right)\left(D_{\tau} \varphi_{n}\right)
$$

together with the condition that the constant term vanishes:

$$
\varphi_{m, n}=O(q)
$$

Since $\varphi_{m}$ is odd in $m$, the definition implies the symmetries

$$
\forall m, n: \varphi_{m, n}=\varphi_{n, m}=\varphi_{-m,-n} .
$$

Moreover, $\varphi_{m, 0}=0$ as $\varphi_{0}=0$. Our second main result describes the modular properties of $\varphi_{m, n}$ :

Theorem 1.3 For all $m, n \in \mathbb{Z}$ the difference

$$
\varphi_{m, n}-|n| \delta_{m+n, 0}
$$

is a quasi-Jacobi form of weight 0 and index $\frac{1}{2}(|m|+|n|)$.

\footnotetext{
1 For any $a \in \mathbb{Q}$ the ratio $\frac{\Theta(x+a)}{\Theta(x)}$ is a meromorphic Jacobi form of index 0 (of higher level). Hence all its Taylor coefficients are modular forms.
} 
If $m \neq-n$ the proof of Theorem 1.3 is easy. Indeed, in this case we have

$$
\varphi_{m, n}=\frac{m}{m+n} \varphi_{m} D_{\tau}\left(\varphi_{n}\right)+\frac{n}{m+n} D_{\tau}\left(\varphi_{m}\right) \varphi_{n}
$$

and since the algebra of quasi-Jacobi forms is closed under differentiation with respect to both $z$ and $\tau$ the result follows from Theorem 1.2. It hence remains to consider the case $m=-n$. However, since the algebra of quasi-Jacobi forms is not closed under integration, this case is not obvious at all.

A key feature of the functions $\varphi_{m}$ is their polynomial dependence on $m$. Precisely, their Taylor expansion in the elliptic variable is of the form

$$
\varphi_{m}=\sum_{k \geq 1} P_{k}(m) z^{k}
$$

where each $P_{k}$ is a polynomial in $m$ of degree $\leq k$ with coefficients quasi-modular forms. This implies that the $\varphi_{m, n}$ depend polynomially on $m, n$ as well. Hence we are allowed to take the limit of the formula (6). The result is

$$
\varphi_{n,-n}=D_{\tau}\left(\varphi_{n}\right) \varphi_{-n}+n\left(D_{\tau}\left(\varphi_{-n}^{\prime}\right) \varphi_{n}-\varphi_{-n}^{\prime} D_{\tau} \varphi_{n}\right)
$$

where $\varphi_{u}^{\prime}$ is the formal derivative of $\varphi_{u}$ with respect to $u$. But, by inspection the function $\varphi_{n}^{\prime}$ is usually not a quasi-Jacobi and hence from this point it is still unclear why $\varphi_{n,-n}$ should be quasi-Jacobi. Instead our proof of Theorem 1.3 relies on a subtle interplay between holomorphic anomaly equations, which measure the defect of $\varphi_{m}$ and $\varphi_{m, n}$ to be honest Jacobi forms, and the aforementioned polynomiality.

The holomorphic anomaly equations we derive are also of independent interest since they determine the precise transformation behaviour of the functions $\varphi_{m}$ and $\varphi_{m, n}$ under the Jacobi group. As another indirect consequence of the proof of Theorem 1.3 we obtain a third, recursive characterization of the function $\varphi_{m}$ :

Proposition 1.4 For all $m, n \geq 1$ we have

$$
\varphi_{m+n}=\frac{1}{m} D_{z}\left(\varphi_{m}\right) \varphi_{n}+\frac{1}{n} \varphi_{m} D_{z}\left(\varphi_{n}\right)+\sum_{i+j=m} \frac{1}{i} \varphi_{i, n} \varphi_{j}+\sum_{i+j=n} \frac{1}{i} \varphi_{i, m} \varphi_{j} .
$$

We finally relate the functions $\varphi_{m}$ and $\varphi_{m, n}$ to the geometry of $\mathrm{K} 3$ surfaces.

Conjecture 1.5 The functions $\varphi_{m}$ and $\varphi_{m, n}$ as defined above are the functions appearing in Conjecture 1.1.

Besides plenty of evidence which is known for Conjecture 1.1, e.g. [18], there are several qualitative features of the $\varphi$ 's which correspond to similar features in Gromov-Witten theory. The polynomial dependence is reflected in the polynomial dependence of the double ramification cycle on the ramification profiles [6]. In their Taylor expansions the $z$-coefficients of the $\varphi$ 's are quasi-modular forms. This matches a 
result of [12]. The quasi-Jacobi form property and the holomorphic anomaly equations are expected from holomorphic-symplectic geometry [15] and the results of [17].

Conjecture 1.1 yields an explicit formula for the Gromov-Witten theory of $K 3 \times \mathbb{P}^{1}$ relative to two fibers over $\mathbb{P}^{1}$. In terms of this theory, efficient algorithms to determine the Gromov-Witten invariants of all CHL Calabi-Yau threefolds are known [1]. This leads to deep relations between counting on K3 surfaces and Conway moonshine. We hope to come back to these questions in future work.

\section{Preliminaries}

\subsection{Quasi-modular forms}

For all even $k>0$ consider the renormalized Eisenstein series

$$
G_{k}(\tau)=-\frac{B_{k}}{2 \cdot k}+\sum_{n \geq 1} \sum_{d \mid n} d^{k-1} q^{n} .
$$

The $\mathbb{C}$-algebras Mod $=\oplus_{k} \operatorname{Mod}_{k}$ and QMod $=\oplus_{k} \mathrm{QMod}_{k}$ of modular and quasimodular forms can be described by Eisenstein series:

$$
\operatorname{Mod}=\mathbb{C}\left[G_{4}, G_{6}\right], \quad \text { QMod }=\mathbb{C}\left[G_{2}, G_{4}, G_{6}\right]
$$

The algebra QMod is acted on by both $D_{\tau}=q \frac{d}{d q}$ and the operator $\frac{d}{d G_{2}}$ which takes the formal derivative in $G_{2}$ when a quasi-modular forms is written as a polynomial in $G_{2}, G_{4}, G_{6}$. Let also wt be the operator on QMod that acts on QMod $k$ by multiplication by $k$. We have the $\mathrm{sl}_{2}$-commutation relation

$$
\left[\frac{d}{d G_{2}}, D_{\tau}\right]=-2 \mathrm{wt}
$$

\subsection{Theta functions}

Let $z \in \mathbb{C}$ and $p=e^{z}$. Let

$$
\vartheta_{1}(z, \tau)=\sum_{v \in \mathbb{Z}+\frac{1}{2}}(-1)^{\lfloor v\rfloor} p^{v} q^{v^{2} / 2}
$$

be the odd Jacobi theta function. ${ }^{2}$ By the Jacobi triple product we have

$$
\Theta(z)=\vartheta_{1}(z, \tau) / \eta^{3}(\tau)
$$

\footnotetext{
2 The Jacobi function $\vartheta_{1}$ defines the unique section on the elliptic curve $\mathbb{C}_{w} /(\mathbb{Z}+\tau \mathbb{Z})$ which vanishes at the origin. In our convention the variable $w$ of the complex plane $\mathbb{C}_{w}$ is related to $z$ by $z=2 \pi i w$. In other words, the fundamental region of the curve is given by $\frac{z}{2 \pi i} \in\{a+b \tau \mid a, b \in[0,1]\}$.
} 
The product formula for $\Theta$ yields also the expansion

$$
\Theta(z)=z \exp \left(-2 \sum_{k \geq 2} G_{k} \frac{z^{k}}{k !}\right)
$$

\subsection{Quasi-Jacobi forms}

Jacobi forms are a generalization of classical modular forms which depend on an elliptic parameter $z \in \mathbb{C}$ and a modular parameter $\tau \in \mathbb{H}$, see [4] for an introduction. Quasi-Jacobi forms are constant terms of almost holomorphic Jacobi forms. Following [11] and [14, Sec.1] we shortly recall the definition.

Consider the real-analytic functions

$$
\nu=\frac{1}{8 \pi \Im(\tau)}, \quad \alpha=\frac{\Im(z / 2 \pi i)}{\Im(\tau)} .
$$

An almost holomorphic function on $\mathbb{C} \times \mathbb{H}$ is a function of the form

$$
\Psi=\sum_{i, j \geq 0} \psi_{i, j}(z, \tau) v^{i} \alpha^{j}
$$

such that each of the finitely many non-zero $\psi_{i, j}$ is holomorphic and admits a Fourier expansion of the form $\sum_{n>0} \sum_{r \in \mathbb{Z}} c(n, r) q^{n} p^{r}$ in the region $|q|<1$. An almost holomorphic weak Jacobi form of weight $k$ and index $m \in \mathbb{Z}$ is an almost holomorphic function on $\mathbb{C} \times \mathbb{H}$ which satisfies the transformations laws of Jacobi forms of this weight and index [4]. A quasi-Jacobi form of weight $k$ and index $m$ is a function $\psi(z, \tau)$ such that there exists an almost holomorphic weak Jacobi form $\sum_{i, j} \psi_{i, j} v^{i} \alpha^{j}$ with $\psi_{0,0}=\psi$.

In this paper we will also work with quasi-Jacobi forms of half-integral index $\frac{m}{2} \in \frac{1}{2} \mathbb{Z}$. These are defined identical as above except that we include (in the usual way) a character in the required transformation law. The character we use for index $m / 2$ is defined by the transformation properties of $\Theta^{m}(z)$ under the Jacobi group. ${ }^{3}$ In particular, $\Theta(z)$ is a (quasi) Jacobi form of weight -1 and index $1 / 2$; its square $\Theta(z)^{2}$ is a Jacobi form without character.

The algebra of quasi-Jacobi forms is bigraded by weight $k$ and index $m$ :

$$
\mathrm{QJac}=\bigoplus_{k} \bigoplus_{m \in \frac{1}{2} \mathbb{Z}} \mathrm{QJac}_{k, m}
$$

In index 0 we recover the algebra of quasi-modular forms: $\mathrm{QJac}_{k, 0}=\mathrm{QMod}_{k}$.

\footnotetext{
3 This character is essentially uniquely determined by requiring that the square of a half-integral weight Jacobi form is a Jacobi form without character, see for example the discussion in [5].
} 
Similar to the case of quasi-modular forms, the algebra of quasi-Jacobi forms can be embedded in a polynomial algebra. Let $D_{z}=\frac{d}{d z}=p \frac{d}{d p}$ and consider the series

$$
\mathrm{A}(z)=\frac{D_{z} \Theta(z)}{\Theta(z)}=-\frac{1}{2}-\sum_{m \neq 0} \frac{p^{m}}{1-q^{m}}
$$

and the Weierstraß elliptic function

$$
\wp(z, \tau)=\frac{1}{12}+\frac{p}{(1-p)^{2}}+\sum_{d \geq 1} \sum_{k \mid d} k\left(p^{k}-2+p^{-k}\right) q^{d}
$$

We write $\wp^{\prime}(z, \tau)=D_{z} \wp(z, \tau)$ for its derivative with respect to $z$. Since taking the derivative with respect to $z$ and $\tau$ preserves the algebra of quasi-Jacobi forms ([14]) it is easy to see that all of these are (meromorphic) quasi-Jacobi forms.

Proposition 2.1 The algebra $\mathcal{R}=\mathbb{C}\left[\Theta, A, G_{2}, \wp, \wp^{\prime}, G_{4}\right]$ is a free polynomial ring, and $\mathrm{QJac}$ is equal to the subring of all polynomials which define holomorphic functions $\mathbb{C} \times \mathbb{H} \rightarrow \mathbb{H}$.

Proof It is immediate that if $f \in \mathcal{R}$ is holomorphic, then it is a quasi-Jacobi form. Conversely, divide any quasi-Jacobi form of index $m / 2$ by $\Theta^{m}$. The result then follows from [11, Sec. 2].

Remark 1 The algebra $\mathcal{R}$ is the algebra of all meromorphic quasi-Jacobi forms with the property that all poles are at the lattice points $z=m+n \tau$ with $m, n \in \mathbb{Z}$. Indeed, since $\Theta, A$ and $G_{2}$ lie in $\mathcal{R}$, it suffices to show that meromorphic Jacobi forms of index 0 with the latter property are elements of $\mathcal{R}$. For such a Jacobi form there exists a polynomial in $\wp$ and $\wp^{\prime}$ with modular coefficients such that the sum is holomorphic and elliptic, hence constant. Therefore, every such meromorphic Jacobi form lies in $\mathcal{R}$. $\triangle$

The weight and index of the generators of $\mathcal{R}$ are given as follows:

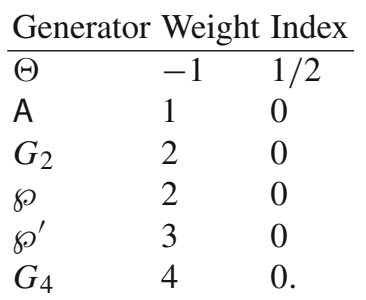

Consider the formal derivative operators $\frac{d}{d \mathrm{~A}}$ and $\frac{d}{d G_{2}}$. Let wt and ind be the operators which act on $\mathrm{QJac}_{k, m}$ by multiplication by the weight $k$ and the index $m$ respectively. 
By $[14,(12)]$ we have the commutation relations:

$$
\begin{array}{ll}
{\left[\frac{d}{d G_{2}}, D_{\tau}\right]=-2 \mathrm{wt},} & {\left[\frac{d}{d \mathrm{~A}}, D_{z}\right]=2 \mathrm{ind}} \\
{\left[\frac{d}{d G_{2}}, D_{z}\right]=-2 \frac{d}{d A},} & {\left[\frac{d}{d A}, D_{\tau}\right]=D_{z} .}
\end{array}
$$

The almost-holomorphic Jacobi forms completing $\mathrm{A}$ and $G_{2}$ are given by

$$
\widehat{\mathrm{A}}=\mathrm{A}+\alpha, \quad \widehat{G_{2}}=G_{2}+v .
$$

Moreover all other generators of $\mathcal{R}$ are (meromorphic) Jacobi forms. Hence the formal derivatives $\frac{d}{d \mathrm{~A}}$ and $\frac{d}{d G_{2}}$ of a quasi-Jacobi form measure the dependence of its completion on the non-holomorphic variables $\alpha$ and $v$, or in other words the failure of a quasi-Jacobi forms to be an honest Jacobi forms. For a quasi-Jacobi form we call $\frac{d}{d A} \psi$ its holomorphic anomaly. An equation of the form $\left(\frac{d}{d A} \psi=\ldots\right)$ will be called a holomorphic anomaly equation. Similar definitions apply to $\frac{d}{d G_{2}}$.

As explained in [14] knowing the holomorphic-anomaly equations of a quasi-Jacobi form is equivalent to knowing their transformation properties unter the Jacobi group. Concretely, we have the following (the case of half-integral index is similar):

Lemma 2.2 ([14]) Let $\psi(z, \tau) \in \mathrm{QJac}_{k, m}$ with $m \in \mathbb{Z}$. Then

$$
\begin{aligned}
\psi\left(\frac{z}{c \tau+d}, \frac{a \tau+b}{c \tau+d}\right)= & (c \tau+d)^{k} e\left(\frac{c m(z / 2 \pi i)^{2}}{c \tau+d}\right) \exp \\
& \left(-\frac{c \frac{d}{d G_{2}}}{4 \pi i(c \tau+d)}+\frac{c \frac{z}{2 \pi i} \frac{d}{d A}}{c \tau+d}\right) \psi(z, \tau) \\
\psi(z+2 \pi i(\lambda \tau+\mu), \tau)= & e\left(-m \lambda^{2} \tau-2 \lambda m \frac{z}{2 \pi i}\right) \exp \left(-\lambda \frac{d}{d A}\right) \psi(z, \tau),
\end{aligned}
$$

for all $\gamma=\left(\begin{array}{ll}a & b \\ c & d\end{array}\right) \in \mathrm{SL}_{2}(\mathbb{Z})$ and $(\lambda, \mu) \in \mathbb{Z}^{2}$, where we write e $(x)$ for $\exp (2 \pi i x)$.

\subsection{Multivariate quasi-Jacobi forms}

As in [14, Sec. 2] one can similarly define quasi-Jacobi forms of rank $n$ in which the dependence of the variable $z \in \mathbb{C}$ is generalized to a dependence on the vector

$$
z=\left(z_{1}, \ldots, z_{n}\right) \in \mathbb{C}^{n}
$$

The index of quasi-Jacobi forms of rank $n$ is given by a symmetric matrix

$$
m=\left(\begin{array}{ccc}
m_{11} & \cdots & m_{1 n} \\
\vdots & \ddots & \vdots \\
m_{n 1} & \cdots & n_{n n}
\end{array}\right) .
$$


Although a description of the algebra QJac ${ }^{(n)}$ of rank $n$ quasi-Jacobi forms in terms of concrete polynomial rings is not available in general, using the expansions (9) shows that we have an embedding

$$
\mathrm{QJac}^{(n)} \subset \operatorname{MJac}^{(n)}\left[G_{2}, \mathrm{~A}\left(z_{1}\right), \ldots, \mathrm{A}\left(z_{n}\right)\right]
$$

where we let $\mathrm{MJac}^{(n)}$ denote the algebra of meromorphic-Jacobi forms of rank $n$. In particular, the formal derivative operators

$$
\frac{d}{d \mathrm{~A}\left(z_{i}\right)}, \quad \frac{d}{d G_{2}}
$$

are well-defined. ${ }^{4}$ By $[14,(12)]$ the operators satisfy the commutation relations

$$
\begin{aligned}
& {\left[\frac{d}{d G_{2}}, D_{\tau}\right]=-2 \mathrm{wt}, \quad\left[\frac{d}{d A\left(z_{i}\right)}, D_{z_{j}}\right]=2 \text { ind }_{i, j},} \\
& {\left[\frac{d}{d G_{2}}, D_{z_{i}}\right]=-2 \frac{d}{d \mathrm{~A}\left(z_{i}\right)}, \quad\left[\frac{d}{d A\left(z_{i}\right)}, D_{\tau}\right]=D_{z_{i}} \text {, }}
\end{aligned}
$$

where the operator ind ${ }_{i, j}$ multiplies a quasi-Jacobi form of index $m$ by $m_{i j}$.

\subsection{Polynomiality}

The following simple lemma about polynomials will be convenient for us later.

Lemma 2.3 Let $f(u, v)$ be a polynomial in variables $u, v$ and let $F(u)$ be the unique polynomial such that $\forall n \geq 1: F(n)=\sum_{j=0}^{n-1} f(j, n-j)$. Then

$$
F(-n)=-\sum_{j=1}^{n} f(-j,-n+j)
$$

Proof For all $m \in \mathbb{Z}, n>0$ define

$$
G(m, n)=\sum_{j=0}^{n-1} f(j, m-j) .
$$

This agrees with a unique polynomial $P(m, n)$. Now extend $G$ to all $m, n \in \mathbb{Z}$ by setting $G(m, 0)=0$ and

$$
G(m, n)=-\sum_{j=1}^{-n} G(-j, m+j)
$$

\footnotetext{
4 See also [14] for a direct definition via the almost-holomorphic completions.
} 
for all $m \in \mathbb{Z}, n<0$. We then have that $G(m, n+1)-G(m, n)=f(n, m-n)$ is a polynomial for all $m, n$. But $P(m, n+1)-P(m, n)$ is also a polynomial. The two polynomials agree for $n>0$, so they agree for all $n$; since $G$ and $P$ also agree for $n>0$, this means that they must also agree for all $n$.

The lemma now follows, since it is just saying that $F(-n)=P(-n,-n)=$ $G(-n,-n)$.

We also will find the following language convenient: we say that a set of power series $f_{m}(z) \in R[[z]], m \in \mathbb{Z}$ for some coefficient ring $R$ is polynomial in $m$ if there exist polynomials $P_{k}(u) \in R[u]$ such that

$$
\forall m \in \mathbb{Z}: f_{m}(z)=\sum_{k \geq 0} P_{k}(m) z^{k}
$$

In our case the coefficient ring $R$ will usually be the ring of quasi-modular forms QMod.

\section{Differential equation}

In this section we study the function $\varphi_{m}$ defined by the differential equation (1) and the constant term $\varphi_{m}=p^{m / 2}-p^{-m / 2}+O(q)$. We first prove the evaluation

$$
\varphi_{m}=\operatorname{Res}_{x=0}\left(\frac{\Theta(x+z)}{\Theta(x)}\right)^{m}
$$

which immediately implies that $\varphi_{m}$ is a quasi-Jacobi form. We then study the Fourier expansion of $\varphi_{m}$, discuss the dependence of $\varphi_{m}$ on the parameter $m$, and derive a holomorphic anomaly equation.

\subsection{Proof of Theorem 1.2}

Define functions $\varphi_{m}, m \geq 0$ by the claim of the theorem i.e. let $\varphi_{m}=$ $\operatorname{Res}_{x=0}\left(\frac{\Theta(x+z)}{\Theta(x)}\right)^{m}$. We need to check that these function satisfy the differential equations (1) and have the right constant term (2). Checking the constant term is straightforward and we omit the details (see also Sect. 3.2). To check the differential equation we form the generating series $g(y)=\sum_{m \geq 1} y^{m} \varphi_{m} / m$. Let also $D_{y}=y \frac{d}{d y}$. The differential equation (1) is then equivalent to

$$
D_{\tau}^{2} g(y)=F(z, \tau) D_{y}^{2} g(y) .
$$

Consider the function $f(x)=\frac{\Theta(x+z)}{\Theta(x)}$ and apply the variable change

$$
y=\frac{1}{f(x)} \Longleftrightarrow x=g(y)
$$


where we have used Lagrange inversion to identify the inverse of $1 / f$ with the generating series $g(y)$. Let $f^{\prime}(x):=D_{x} f:=\frac{d}{d x} f(x)$. By differentiating $f(g(y))=y$ and applying the chain rule we find the transformations

$$
\begin{aligned}
& D_{y} g(y)=-\frac{f}{f^{\prime}}, \quad D_{\tau} g(y)=-\frac{D_{\tau} f}{f^{\prime}}, \quad D_{y}^{2} g(y)=-\frac{f}{f^{\prime}} \cdot \frac{f^{\prime \prime} f-\left(f^{\prime}\right)^{2}}{\left(f^{\prime}\right)^{2}}, \\
& D_{\tau}^{2} g(y)=-\frac{1}{\left(f^{\prime}\right)^{3}}\left[D_{\tau}^{2}(f)\left(f^{\prime}\right)^{2}-2 f^{\prime} \cdot D_{\tau}(f) D_{\tau}\left(f^{\prime}\right)+f^{\prime \prime} \cdot D_{\tau}(f)^{2}\right] .
\end{aligned}
$$

Applying these and changing variables the differential equation (11) becomes

$$
D_{x}(f)^{2} D_{\tau}^{2}(f)-2 D_{x}(f) D_{x} D_{\tau}(f) D_{\tau}(f)+D_{x}^{2}(f) D_{\tau}(f)^{2}=F(z, \tau) \cdot D_{x}^{2} \log (f) \cdot f^{3} .
$$

The functions $\Theta(x+z)$ and $\Theta(x)$ are Jacobi forms of rank 2 in the elliptic variables $(x, z)$ of index $\frac{1}{2}\left(\begin{array}{ll}1 & 1 \\ 1 & 1\end{array}\right)$ and $\left(\begin{array}{cc}1 / 2 & 0 \\ 0 & 0\end{array}\right)$ respectively. Hence $f(x)$ is a Jacobi form of weight 0 and index

$$
\left(\begin{array}{cc}
0 & 1 / 2 \\
1 / 2 & 1 / 2
\end{array}\right)
$$

We need to show that the following function vanishes:

$$
\begin{aligned}
\mathcal{F}(x, z)= & D_{x}(f)^{2} D_{\tau}^{2}(f)-2 D_{x}(f) D_{x} D_{\tau}(f) D_{\tau}(f)+D_{x}^{2}(f) D_{\tau}(f)^{2} \\
& -F(z, \tau) \cdot D_{x}^{2} \log (f) \cdot f^{3}
\end{aligned}
$$

As a polynomial in the derivatives of $f$, the function $\mathcal{F}$ is a rank 2 quasi-Jacobi form of weight 6 and index $\frac{1}{2}\left(\begin{array}{ll}0 & 3 \\ 3 & 3\end{array}\right)$. Using the commutation relations (10) a direct check shows

$$
\frac{d}{d G_{2}} \mathcal{F}=\frac{d}{d A(x)} \mathcal{F}=0 .
$$

In particular, by $\left[14\right.$, Lem. 6] we have $\mathcal{F}(x+2 \pi i \tau, z)=p^{-3} \mathcal{F}(x, z)$. Moreover, by considering the Taylor expansion one checks (e.g. using a computer ${ }^{5}$ ) that $\mathcal{F}$ is holomorphic at $x=0$ and vanishes to order 3 at $x=-z$ (use the variable change $\tilde{x}=$ $x+z$ ). We conclude that the ratio $\mathcal{F} / f^{3}$ is a doubly periodic and holomorphic in $x$, so a constant in $x$. The constant is a quasi-Jacobi form in $z$ and is easily checked to vanish. This shows that the differential equation is satisfied. The claim that the $\varphi_{m}$ are quasi-Jacobi forms of the specified weight follows from Lemma 3.1 below.

Define the operator on the algebra of quasi-Jacobi forms by

$$
D=D_{z}+2 G_{2} \frac{d}{d \mathrm{~A}}
$$

\footnotetext{
5 The code for this computation as well as a parallel computation in Sect. 4.2 can be found on the webpage of the second author. It also contains functions which express the $\varphi_{m}, \varphi_{m n}$ in terms of the generators of $\mathcal{R}$.
} 
We conclude the following structure result.

Lemma 3.1 For every $m \geq 0$ there exist modular forms $h_{k} \in \operatorname{Mod}_{m-k-1}$ such that

$$
\varphi_{m}=\sum_{k=0}^{m-1} h_{k}(\tau) \cdot D^{k}\left(\Theta(z)^{m}\right) .
$$

Hence every $\varphi_{m}$ is a quasi-Jacobi form of weight -1 and index $\frac{|m|}{2}$, and $\frac{d}{d G_{2}} \varphi_{m}=0$.

Proof of Corollary For any power series $f(z)$ we have

$$
e^{D_{z} x} f(z)=f(x+z)
$$

Moreover, the Baker-Campbell-Hausdorf formula and the relations (8) yield

$$
e^{D_{z} x} e^{2 G_{2} \frac{d}{d \mathrm{~A}} x}=e^{x D-2 x^{2} G_{2} \text { ind }}=e^{-2 x^{2} G_{2} \text { ind }} e^{x D} .
$$

We find that

$$
\begin{aligned}
\frac{\Theta(x+z)^{m}}{\Theta(x)^{m}} & =\Theta(x)^{-m} e^{D_{z} x}\left(\Theta(z)^{m}\right) \\
& =\Theta(x)^{-m} e^{D_{z} x} e^{2 G_{2} \frac{d}{d \mathrm{~A}} x}\left(\Theta(z)^{m}\right) \\
& \stackrel{(13)}{=} \Theta(x)^{-m} e^{-m x^{2} G_{2}} e^{x D}\left(\Theta(z)^{m}\right) \\
& =x^{-m} \exp \left(2 m \sum_{k \geq 4} G_{k} \frac{x^{k}}{k !}\right) e^{x D}\left(\Theta(z)^{m}\right)
\end{aligned}
$$

where we used (7) in the last step. Taking the coefficient of $x^{-1}$ yields the first claim. The second claim follows from the commutation relation $\left[\frac{d}{d G_{2}}, D\right]=0$.

Remark 2 For all $m \geq 0$ we have

$$
\varphi_{-m}=\operatorname{Res}_{x=-z}\left(\frac{\Theta(x+z)}{\Theta(x)}\right)^{-m}
$$

Indeed, after the variable change $x^{\prime}=-(x+z)$ the right hand side becomes

$$
-\operatorname{Res}_{x^{\prime}=0}\left(\frac{\Theta\left(-x^{\prime}-z\right)}{\Theta\left(-x^{\prime}\right)}\right)^{m}=-\varphi_{m} .
$$




\subsection{Fourier expansion}

By integrating the function

$$
f_{m}(x)=\left(\frac{\Theta(x+z)}{\Theta(x)}\right)^{m}
$$

around the sides of a fundamental region and using $f_{m}(x+\tau, z)=p^{-m} f_{m}(x, z)$ one gets

$$
\varphi_{m}=\operatorname{Res}_{x=0} f_{m}=\left(1-p^{-m}\right) \operatorname{Coeff}_{\sigma^{0}} f_{m}(x, z, \tau)
$$

where $\sigma=e^{x}$ is the Fourier variable associated to $x .^{6}$

An application of the Jacobi triple product and computing the power by $m$ by taking first the log of each product term, multiplying it by $m$ and then exponentiating again, together with a bit of reordering the terms, then yields from this the expression

$$
\begin{aligned}
\varphi_{m} & =\left(p^{m / 2}-p^{-m / 2}\right) \operatorname{Coeff}_{\sigma^{0}} \exp \left(\sum_{k \neq 0} \frac{m}{k} \sigma^{k} \frac{1-p^{k}}{1-q^{k}}\right) \\
& =\left(p^{m / 2}-p^{-m / 2}\right) \sum_{|a|=0}\left(\prod_{i} \frac{1-p^{a_{i}}}{1-q^{a_{i}}}\right) \frac{m^{l(a)}}{\mathfrak{z}(a)}
\end{aligned}
$$

where the sum in the second equation is over all generalized partitions with non-zero parts summing up to 0 . Moreover, if we write $a=\left(i^{a_{i}}\right)_{i \in \mathbb{Z} \backslash\{0\}}$ then $\mathfrak{z}(a)=\prod_{i} i^{a_{i}} a_{i}$ ! is the standard automorphism factor. The first Fourier coefficients of $\varphi_{m}$ are

$$
\varphi_{m}=\left(s^{m}-s^{-m}\right)\left(1-m^{2}\left(s-s^{-1}\right)^{2} q+O\left(q^{2}\right)\right)
$$

where we have written $s=e^{z / 2}$ so $p=s^{2}$.

\subsection{The solution $\phi_{m}$ as a function of $m$}

In this section we consider $\varphi_{m}$ as a function of $m$ viewed as a (formal) variable. To distinguish with the case $m \in \mathbb{Z}$ we will replace $m$ by a variable $u$.

We give three different formulas for $\varphi_{u}$. First, consider the expansion

$$
F(s, q)=\sum_{k \geq 1} F_{k}(s) q^{k}, \quad F_{k}(s)=-\sum_{d \mid k}\left(\frac{k}{d}\right)^{3}\left(s^{d}-s^{-d}\right)^{2}
$$

where as before we have used $s=e^{z / 2}$ so $p=s^{2}$. Then by an immediate check the differential equation (1) for $\varphi_{m}$ is equivalent to the following formula:

\footnotetext{
6 See also [17, App. A] for a similar argument.
} 
$\varphi_{u}=\left(p^{u / 2}-p^{-u / 2}\right)\left(1+\sum_{r \geq 1} \sum_{k_{1}, \ldots, k_{r} \geq 1} \frac{F_{k_{1}}(s) F_{k_{2}}(s) \cdots F_{k_{r}}(s)}{k_{1}^{2}\left(k_{1}+k_{2}\right)^{2} \ldots\left(k_{1}+\ldots+k_{r}\right)^{2}} q^{k_{1}+\ldots+k_{r}} u^{2 r}\right)$.

Second we can use the Fourier expansion of the $\varphi_{m}$ as discussed in Sect. 3.2:

$$
\varphi_{u}=\left(p^{u / 2}-p^{-u / 2}\right) \sum_{|a|=0}\left(\prod_{i} \frac{1-p^{a_{i}}}{1-q^{a_{i}}}\right) \frac{u^{l(a)}}{\mathfrak{z}(a)}
$$

where we used that (15) makes sense for arbitrary $u$. We see that Theorem 1.2 is equivalent to the following non-trivial identity:

$$
\sum_{|a|=0}\left(\prod_{i} \frac{1-p^{a_{i}}}{1-q^{a_{i}}}\right) \frac{u^{l(a)}}{\mathfrak{z}(a)}=1+\sum_{\substack{r \geq 1 \\ k_{1}, \ldots, k_{r} \geq 1}} \frac{F_{k_{1}}(s) F_{k_{2}}(s) \cdots F_{k_{r}}(s)}{k_{1}^{2}\left(k_{1}+k_{2}\right)^{2} \ldots\left(k_{1}+\ldots+k_{r}\right)^{2}} q^{k_{1}+\ldots+k_{r}} u^{2 r}
$$

For the third formula, we use a Taylor expansion in $u$. For positive integers $u$ one can write the solution

$$
\operatorname{Coeff}_{x^{-1}}\left(\frac{\Theta(x+z)}{\Theta(x)}\right)^{u}
$$

as

$$
\begin{aligned}
\varphi_{u} & =\operatorname{Coeff}_{x^{-1}} \frac{(x+z)^{u}}{x^{u}} \exp \left(2 u \sum_{k \geq 2} G_{k} \frac{x^{k}-(x+z)^{k}}{k !}\right) \\
& =\operatorname{Coeff}_{x^{-1}} \sum_{\ell=1}^{\infty}\left(\begin{array}{l}
u \\
\ell
\end{array}\right)\left(\frac{z}{x}\right)^{\ell} \exp \left(2 u \sum_{k \geq 2} G_{k} \frac{x^{k}-(x+z)^{k}}{k !}\right) .
\end{aligned}
$$

The latter expression makes sense as an element of $\mathbb{C}[[z]]$ for all $u \in \mathbb{C}$. For example, the first terms read

$$
\varphi_{u}=u z-G_{2} u^{3} z^{3}+\left(\left(\frac{1}{3} G_{2}^{2}-\frac{1}{72} G_{4}\right) u^{5}+\left(\frac{1}{6} G_{2}^{2}-\frac{5}{72} G_{4}\right) u^{3}\right) z^{5}+O\left(z^{7}\right) .
$$

The expansion (17) yields the following important structure result.

Proposition 3.2 For every $k \geq 1$ there exist odd polynomials $P_{k}(u)$ of degree $\leq k$ with coefficients in $\mathrm{QMod}_{k-1}$ such that for all $m \in \mathbb{Z}$

$$
\varphi_{m}=\sum_{o d d k \geq 1} z^{k} P_{k}(m) .
$$


Moreover, $P_{1}(u)=u$ and if $k \geq 2$, then $u^{3} \mid P_{k}(u)$.

\subsection{Anomaly equation}

We consider the holomorphic anomaly of $\varphi_{m}$ with respect to the variable $z$.

Proposition 3.3 For all $m \geq 1$ one has

$$
\frac{d}{d A} \varphi_{m}=\frac{1}{2} \sum_{\substack{i+j=m \\ i, j \geq 1}} \frac{m^{2}}{i j} \varphi_{i} \varphi_{j} .
$$

It follows that every $z^{k}$ coefficient of $\frac{d}{d A} \varphi_{m}$ is polynomial in $m$ in the range $m \geq 0$. However the dependence on $m$ is only piecewise polynomial in general:

Corollary 3.4 The difference

$$
\varphi_{m}^{A}=\frac{d}{d A} \varphi_{m}-m z \varphi_{m} \delta_{m<0}
$$

depends polynomially on $m$, i.e. there exist polynomials $Q_{k}(u)$ of degree $\leq k+1$ with coefficients in QMod $_{k-2}$ such that $\varphi_{m}^{A}=\sum_{k \geq 2} z^{k} Q_{k}(m)$. Moreover, $u^{2} \mid Q_{k}$ for all $k$.

Proof of Corollary 3.4 We first rewrite the proposition as

$$
\frac{d}{d A} \varphi_{m}=m \sum_{j=1}^{m-1} \varphi_{j} \cdot \frac{\varphi_{m-j}}{m-j}
$$

Hence for all $m \geq 0$ we have $\frac{d}{d A} \varphi_{m}=\sum_{n} Q_{n}(m) z^{n}$ where the polynomials $Q_{n}$ are determined by

$$
Q_{n}(m)=m \sum_{\substack{k+\ell=n \\ k, \ell \geq 1}} \sum_{j=1}^{m-1} P_{k}(j) \frac{P_{\ell}(m-j)}{m-j}
$$

for all $m \geq 0$. Here $P_{k}(m)$ are the polynomials of Proposition 3.2.

For all $m>0$ by Lemma 2.3 we have

$$
\begin{aligned}
Q_{n}(-m) & =-\left.(-m) \sum_{\substack{k+\ell=n \\
k, \ell \geq 1}} \sum_{j=1}^{m} P_{k}(-j)\left(\frac{P_{\ell}(u)}{u}\right)\right|_{u=-m+j} \\
& =-m \sum_{\substack{k+\ell=n \\
k, \ell \geq 1}} \sum_{j=1}^{m-1} P_{k}(j) \frac{P_{\ell}(-m+j)}{-m+j}-m P_{n-1}(m),
\end{aligned}
$$


where we used the second part of Proposition 3.2 for the last equality. Summing up we obtain as desired

$$
\varphi_{-m}^{A}=\sum_{n} z^{n} Q_{n}(-m)=-\frac{d}{d A} \varphi_{m}-m z \varphi_{m} .
$$

Proof of Proposition 3.3 We give first a proof via generating series. As in the proof of Theorem 1.2 consider the generating series

$$
g(y)=\sum_{m \geq 1} \frac{\varphi_{m}}{m} y^{m}
$$

and let $D_{y}=y \frac{d}{d y}$. We need to prove the equality

$$
\frac{d}{d \mathrm{~A}(z)} g(y)=g(y) D_{y} g(y)
$$

Let $f(x)=\frac{\Theta(x+z)}{\Theta(x)}$ so that $f(g(y))=\frac{1}{y}$. Then by $\left[\frac{d}{d \mathrm{~A}}, D_{z}\right]=2$ ind we have

$$
\frac{d}{d \mathrm{~A}(z)} f(x)=\frac{d}{d \mathrm{~A}(z)} \frac{e^{D_{z} x} \Theta(z)}{\Theta(x)}=\frac{\left[\frac{d}{d \mathrm{~A}(z)}, D_{z}\right] x e^{D_{z} x} \Theta(z)}{\Theta(x)}=x \cdot f(x) .
$$

Applying $\frac{d}{d A}$ to $f(g(y))=1 / y$ we get $\left(\frac{d}{d A} f\right)(g(y))+\left(D_{x} f\right)(g(y)) \frac{d}{d A} g(y)=0$, and hence

$$
\frac{d}{d \mathrm{~A}(z)} g(y)=-\frac{g(y)}{y \cdot\left(D_{x} f\right)}
$$

Since we also have

$$
D_{y}(f(g(y)))=\left(D_{x} f\right)(g(y)) D_{y}(g(y))=-\frac{1}{y}, \text { and hence } \frac{1}{D_{x} f}=-y \cdot D_{y} g(y)
$$

the claim follows.

We give a more direct proof of Proposition 3.3 using the following combinatorial Lemma whose proof follows directly from Lagrange inversion and is left to the reader.

Lemma 3.5 Let $f(x)$ be a power series and $k \in \mathbb{N}$. Then for all $m \geq 1$ we have

$$
\frac{1}{m} \cdot\left[f(x)^{m}\right]_{x^{m-k}}=\frac{1}{k} \sum_{n_{1}+\ldots+n_{k}=m} \prod_{i=1}^{k} \frac{1}{n_{i}}\left[f(x)^{n_{i}}\right]_{x^{n_{i}-1}}
$$

where we write $[-]_{x^{m}}$ for taking the coefficient of $x^{m}$. 
Second proof of Proposition 3.3 Observe that by (18) we have

$$
\frac{d}{d A}\left[\left(\frac{\Theta(x+z)}{\Theta(x)}\right)^{m}\right]_{x^{-1}}=m\left[\left(\frac{\Theta(x+z)}{\Theta(x)}\right)^{m}\right]_{x^{-2}},
$$

Applying Lemma 3.5 with $k=2$ and $f=x \frac{\Theta(x+z)}{\Theta(x)}$ yields the desired result.

\section{Differential equation of the second kind}

Recall the two defining properties of the series $\varphi_{m, n}$ :

- the differential equation: $D_{\tau} \varphi_{m, n}=m n \varphi_{m} \varphi_{n} F+\left(D_{\tau} \varphi_{m}\right)\left(D_{\tau} \varphi_{n}\right)$

- the vanishing of the constant term: $\varphi_{m, n}=O(q)$.

The goal of this section is to first prove that $\varphi_{m, n}$ are quasi-Jacobi forms (Theorem 1.3), and then derive their holomorphic anomaly equations (Sect. 4.4).

\subsection{Polynomiality}

We first recall the following.

Proposition 4.1 If $m \neq-n$ then we have

$$
\varphi_{m, n}=\frac{m}{m+n} \varphi_{m} D_{\tau}\left(\varphi_{n}\right)+\frac{n}{m+n} D_{\tau}\left(\varphi_{m}\right) \varphi_{n}
$$

Proof The differential equation follows from the defining differential equation (1) satisfied by $\varphi_{m}$. The vanishing of the constant term is observed directly.

By definition and the polynomiality of $\varphi_{m}$ the series $\varphi_{m, n}$ is a power series in $z$ and $q$ with coefficients which are polynomials in $m$ and $n$. We use Proposition 4.1 to prove a stronger statement.

Proposition 4.2 There exist polynomials $P_{r}(u, v)$ of degree at most $r$ in variables $u, v$ with coefficients quasi-modular forms of weight $r$ such that for all $m, n \in \mathbb{Z}$

$$
\varphi_{m, n}=\sum_{r>0} z^{r} P_{r}(m, n)
$$

Moreover, the polynomials $P_{r}(u, v)$ are divisible by both $u^{2}$ and $v^{2}$.

Proof By the defining differential equation (5) and the polynomiality of $\varphi_{m}$ there exist polynomials $P_{a, r}(u, v)$ of degree $r+2$ with rational coefficients such that

$$
\varphi_{m, n}=\sum_{r>0} z^{r} \sum_{a \geq 1} q^{a} P_{a, r}(m, n)
$$


for all $m, n \in \mathbb{Z}$. Here we have $r>0$ since $\varphi_{m}(z=0)=0$ for all $m$.

On the other hand by Proposition 4.1 for all $m, n \in \mathbb{Z}$ with $m \neq-n$ we have

$$
\varphi_{m, n}=\sum_{r>0} z^{r} \frac{1}{m+n} \sum_{k+\ell=r}\left(n D_{\tau}\left(P_{k}(m)\right) P_{\ell}(n)+m P_{k}(m) D_{\tau}\left(P_{\ell}(n)\right)\right)
$$

where $P_{k}(u)$ are the polynomials of Proposition 3.2. Since the inner sum vanishes when setting $m=-n$ and it is polynomial of degree at most $r+1$ in $m, n$, there exists a polynomial $P_{r}(u, v)$ of degree at most $r$ with coefficients in $\mathrm{QMod}_{r}$ such that

$$
\varphi_{m, n}=\sum_{r>0} z^{r} P_{r}(m, n)
$$

whenever $m \neq-n$.

The equality of polynomials

$$
\sum_{a \geq 1} q^{a} P_{a, r}(u, v)=P_{r}(u, v)
$$

holds after evaluating $(u, v)$ at $(m, n)$ for all integers $m \neq-n$. Hence the equality holds as an equality of polynomials.

The last statement follows since $n D_{\tau}\left(P_{k}(m)\right) P_{\ell}(n)+m P_{k}(m) D_{\tau}\left(P_{\ell}(n)\right)$ is divisible by both $m^{2}$ and $n^{2}$, hence the same holds for the term obtained by dividing by $m+n$.

Example 4.3 The first terms in the Fourier and Taylor expansions of $\varphi_{m, n}$ are

$$
\varphi_{m, n}=-m n\left(s^{m}-s^{-m}\right)\left(s^{n}-s^{-n}\right)\left(s-s^{-1}\right)^{2} q+O\left(q^{2}\right)
$$

where $s=e^{z / 2}$, and

$$
\begin{aligned}
\varphi_{u, v}= & \left(\left(2 G_{2}^{2}-\frac{5}{6} G_{4}\right) u^{2} v^{2}\right) z^{4}+\left(\left(-\frac{4}{3} G_{2}^{3}+\frac{2}{3} G_{2} G_{4}-\frac{7}{720} G_{6}\right)\left(u^{4} v^{2}+u^{2} v^{4}\right)\right. \\
& \left.+\left(-\frac{2}{3} G_{2}^{3}+\frac{1}{6} G_{2} G_{4}+\frac{7}{720} G_{6}\right) u^{3} v^{3}+\left(-\frac{2}{3} G_{2}^{3}+\frac{5}{6} G_{2} G_{4}-\frac{7}{144} G_{6}\right) u^{2} v^{2}\right) z^{6}+O\left(z^{7}\right) .
\end{aligned}
$$

\subsection{Holomorphic anomaly equations}

From Proposition 4.1 we can deduce for all $m \neq-n$ the following anomaly equation:

$$
\begin{aligned}
\frac{d}{d A} \varphi_{m, n}= & \frac{n}{m+n}\left(D_{z}\left(\varphi_{m}\right) \varphi_{n}+D_{\tau}\left(\frac{d}{d A} \varphi_{m}\right) \varphi_{n}+D_{\tau}\left(\varphi_{m}\right) \cdot \frac{d}{d A} \varphi_{n}\right) \\
& +\frac{m}{m+n}\left(\varphi_{m} D_{z}\left(\varphi_{n}\right)+\left(\frac{d}{d A} \varphi_{m}\right) \cdot D_{\tau}\left(\varphi_{n}\right)+\varphi_{m} \cdot D_{\tau} \frac{d}{d A} \varphi_{n}\right)
\end{aligned}
$$


By the anomaly equation for $\varphi_{m}$ this gives an expression for $\frac{d}{d A} \varphi_{m, n}$ whenever $m \neq$ $-n$.

In case $m, n>0$ we can find a more efficient equation:

Proposition 4.4 For all $m, n>0$,

$$
\frac{d}{d A} \varphi_{m, n}=\frac{m \cdot n}{m+n} \varphi_{m+n}+\sum_{j=1}^{m-1} \frac{m}{j} \varphi_{m-j, n} \varphi_{j}+\sum_{j=1}^{n-1} \frac{n}{j} \varphi_{m, n-j} \varphi_{j}
$$

Proof We prove that the right hand side in (20) is equal to the right hand side in (19). By the anomaly equation for $\varphi_{m}$ and comparing terms it is equivalent to prove the following equation for all $m, n \geq 1$ :

$$
\varphi_{m+n}=\frac{1}{m} D_{z}\left(\varphi_{m}\right) \varphi_{n}+\frac{1}{n} \varphi_{m} D_{z}\left(\varphi_{n}\right)+\sum_{i+j=m} \frac{1}{i} \varphi_{i, n} \varphi_{j}+\sum_{i+j=n} \frac{1}{i} \varphi_{i, m} \varphi_{j}
$$

We multiply both sides with $x^{m} y^{n}$ and sum over all $m, n \geq 1$. With $g(x)=$ $\sum_{m \geq 1} x^{m} \varphi_{m} / m$ the equation becomes

$$
\begin{aligned}
\frac{y D_{x} g(x)-x D_{y} g(y)}{x-y}= & D_{z} g(x) \cdot D_{y} g(y)+D_{x} g(x) \cdot D_{z} g(y)+\left(\left(D_{x}+D_{y}\right)^{-1} D_{y} h(x, y)\right) D_{x} g(x) \\
& +\left(\left(D_{x}+D_{y}\right)^{-1} D_{x} h(x, y)\right) D_{y} g(y)
\end{aligned}
$$

where $\left(D_{x}+D_{y}\right)^{-1}$ acts term-wise by multiplying the coefficient of $x^{m} y^{n}$ by $(m+n)^{-1}$ (this is well defined since both $m, n$ are positive for all non-zero coefficients) and we have used $\left(D_{x}+D_{y}\right) \sum_{m, n \geq 1} \frac{\varphi_{m, n}}{m} x^{m} y^{n}=D_{y} h(x, y)$ with

$$
h(x, y)=D_{x} g(x) \cdot D_{\tau} g(y)+D_{\tau} g(x) \cdot D_{y} g(y) .
$$

Rewriting $D_{y}=\left(D_{x}+D_{y}\right)-D_{x}$ we have

$$
\left(D_{x}+D_{y}\right)^{-1} D_{y} h=h-\left(D_{x}+D_{y}\right)^{-1} D_{x} h .
$$

Inserting this the $\left(D_{x}+D_{y}\right)^{-1}$ term factors out and we obtain that (22) is equivalent to

$$
\begin{aligned}
D_{x} h= & \left(D_{x}+D_{y}\right)\left(\frac{1}{D_{y} g(y)-D_{x} g(x)}\right. \\
& \left.\times\left(\frac{y D_{x} g(x)-x D_{y} g(y)}{x-y}-D_{z} g(x) \cdot D_{y} g(y)+D_{x} g(x) \cdot D_{z} g(y)\right)\right)
\end{aligned}
$$


Expanding and using that $\left(D_{x}+D_{y}\right)(y /(x-y))=0$ this is equivalent to

$$
\begin{aligned}
& \left(D_{x}^{2} g(x) \cdot D_{y} g(y)-D_{x} g(x) \cdot D_{y}^{2} g(y)\right) \cdot\left(1+D_{z} g(x)+D_{z} g(y)+h\right) \\
& \quad+\left(D_{y} g(y)-D_{x} g(x)\right) \cdot\left(D_{z}\left(D_{x} g(x) \cdot D_{y} g(y)\right)+D_{x} g(x) D_{y}(h)+D_{y} g(y) D_{x} h\right)=0 .
\end{aligned}
$$

We consider again the function $f(x)=\frac{\Theta(x+z)}{\Theta(x)}$ and apply the variable change

$$
x=\frac{1}{f(\tilde{x})}, y=\frac{1}{f(\tilde{y})} \Longleftrightarrow \tilde{x}=g(x), \tilde{y}=g(y) .
$$

Let us denote $f^{\prime}(x)=\frac{d}{d x} f(x)$. We then have the transformations

$$
\begin{aligned}
D_{x} g(x) & =-\frac{f}{f^{\prime}} & D_{z} g(x) & =-\frac{D_{z} f}{f} \\
D_{x}^{2} g(x) & =-\frac{f}{f^{\prime}} \cdot \frac{f^{\prime \prime} f-\left(f^{\prime}\right)^{2}}{\left(f^{\prime}\right)^{2}} & D_{\tau} g(x) & =-\frac{D_{\tau} f}{f^{\prime}} \\
D_{x} D_{\tau} g(x) & =-\frac{f}{f^{\prime}} \cdot \frac{f^{\prime \prime} D_{\tau}(f)-f^{\prime} D_{\tau}\left(f^{\prime}\right)}{\left(f^{\prime}\right)^{2}}, & D_{x} D_{z} g(x) & =-\frac{f}{f^{\prime}} \cdot \frac{f^{\prime \prime} D_{z}(f)-f^{\prime} D_{z}\left(f^{\prime}\right)}{\left(f^{\prime}\right)^{2}}
\end{aligned}
$$

where on the right hand side we have omitted the argument $\tilde{x}$ in $f$ and its derivatives.

After changing variables and clearing denominators we find that (23) is equivalent to

$$
\left(f^{\prime \prime}(x) f(x) f^{\prime}(y)^{2}-f^{\prime \prime}(y) f(y) f^{\prime}(x)^{2}\right) \cdot C+\left(f(x) f^{\prime}(y)-f^{\prime}(x) f(y)\right) \cdot D=0
$$

where we have written $x, y$ for $\tilde{x}, \tilde{y}$ and

$$
\begin{aligned}
C= & f^{\prime}(x) f^{\prime}(y)-D_{z} f(x) \cdot f^{\prime}(y)-f^{\prime}(x) D_{z} f(y)+f(x) D_{\tau} f(y)+D_{\tau} f(x) \cdot f(y) \\
D= & \left(f^{\prime \prime}(x) D_{z} f(x)-f^{\prime}(x) D_{z} f^{\prime}(x)\right) f^{\prime}(y)^{2} \\
& +\left(f^{\prime \prime}(y) D_{z} f(y)-f^{\prime}(y) D_{z} f^{\prime}(y)\right) f^{\prime}(x)^{2} \\
& -\left(f^{\prime \prime}(x) f(x)-f^{\prime}(x)^{2}\right) f^{\prime}(y) D_{\tau} f(y)-\left(f^{\prime \prime}(x) D_{\tau} f(x)-f^{\prime}(x) D_{\tau} f^{\prime}(x)\right) f(y) f^{\prime}(y) \\
& -\left(f^{\prime \prime}(y) f(y)-f^{\prime}(y)^{2}\right) f^{\prime}(x) D_{\tau} f(x)-\left(f^{\prime \prime}(y) D_{\tau} f(y)-f^{\prime}(y) D_{\tau} f^{\prime}(y)\right) f(x) f^{\prime}(x) .
\end{aligned}
$$

Let $\mathcal{F}(x, y, z, \tau)$ be the left hand side of (24). We need to show that $\mathcal{F}=0$. We will argue as in Sect. 3.1. Since it is a polynomial in derivatives of Jacobi forms the function $\mathcal{F}$ is a quasi-Jacobi form of the three elliptic variables $x, y, z$. It is of weight 6 and index

$$
L=\left(\begin{array}{ccc}
0 & 0 & 3 / 2 \\
0 & 0 & 3 / 2 \\
3 / 2 & 3 / 2 & 3 / 2
\end{array}\right)
$$


A quick check using the commutation relations (10) shows that in the algebra of such quasi-Jacobi forms we have

$$
\frac{d}{d G_{2}} \mathcal{F}=\frac{d}{d A(x)} \mathcal{F}=\frac{d}{d A(y)} \mathcal{F}=0
$$

By a direct check (e.g. using a computer) $\mathcal{F}$ has no poles at $y=0$ and vanishes to order 3 at $y=-z$. Hence the ratio

$$
\frac{\mathcal{F}(x, y)}{f(x)^{3} f(y)^{3}},
$$

is holomorphic in $y$. Since by [14, Lem. 6] it is also 2-periodic, we find that it is constant in $y$. But $\mathcal{F}$ is symmetric in $x$ and $y$ so it is also constant in $x$. By checking that the constant term vanishes we are done.

Remark 3 In the proof we established (21), which is precisely Proposition 1.4.

By Proposition 4.1 for all $m, n>0$ the function $\varphi_{m, n}$ is determined by $\varphi_{m}$ and $\varphi_{n}$. Hence (21) yields recursive formulas for $\varphi_{m}$, and hence provides an alternative definition of the set of functions $\varphi_{m}$ starting from the initial condition $\varphi_{1}=\Theta(z)$. For example, the case $(n, 1)$ yields

$$
\varphi_{n+1}=D_{z}\left(\varphi_{1}\right) \varphi_{n}+\frac{1}{n} \varphi_{1} D_{z}\left(\varphi_{n}\right)+\sum_{i=1}^{n-1} \frac{1}{i} \varphi_{i, 1} \varphi_{n-i}
$$

\subsection{Proof of Theorem 1.3}

We need to show that for all $n \geq 1$ we have

$$
\varphi_{n,-n}-n \in \mathrm{QJac}_{0, n} .
$$

The idea of the proof is to consider the two expressions for $\frac{d}{d A} \varphi_{m, n}$ for positive $m, n$ given by (19) and (20). These terms are equal for $m>0$, and (with minor modifications) they have natural extensions to $m \leq 0$. We will observe that these extensions are both polynomial in $m$ (when fixing $n$ ) up to the same non-polynomial correction term. Hence they are equal for all $m$.

Concretely, let $n>0$ be fixed and let $R(m, n)$ be the right hand side of (19). Then by Corollary 3.4 the sum of $R(m, n)$ and

$$
-m z \delta_{m<0}\left(\frac{n}{m+n} D_{\tau}\left(\varphi_{m}\right) \varphi_{n}+\frac{m}{m+n} \varphi_{m} D_{\tau}\left(\varphi_{n}\right)\right)=-m z \delta_{m<0} \varphi_{m, n}
$$

is polynomial in $m$. We write

$$
\widetilde{R}(m, n)=R(m, n)-m z \varphi_{m, n} \delta_{m<0}
$$


to denote this polynomial function.

We consider now the right hand side of (20) and we want to make sense of it for negative $m$. For all $m \geq 0$, with $m \neq n$ in the second line, define

$$
\begin{aligned}
S(m, n) & :=\frac{m \cdot n}{m+n} \varphi_{m+n}+\sum_{j=1}^{m-1} \frac{m}{j} \varphi_{m-j, n} \varphi_{j}+\sum_{j=1}^{n-1} \frac{n}{j} \varphi_{m, n-j} \varphi_{j} \\
S(-m, n) & :=\frac{-m \cdot n}{-m+n} \varphi_{-m+n}+\sum_{j=1}^{m-1} \frac{m}{j} \varphi_{-m+j, n} \varphi_{j}+\sum_{j=1}^{n-1} \frac{n}{j} \varphi_{-m, n-j} \varphi_{j} .
\end{aligned}
$$

By a direct application of Lemma 2.3 the sum

$$
\widetilde{S}(m, n)=S(m, n)-m z \varphi_{m, n} \delta_{m<0}
$$

is polynomial in $m$.

By Proposition 4.4 we have $R(m, n)=S(m, n)$, hence $\widetilde{R}(m, n)=\widetilde{S}(m, n)$ for all $m>0$. By polynomiality in $m$ we get $\widetilde{R}(m, n)=\widetilde{S}(m, n)$ for all $m \neq-n$. Thus

$$
\forall m \neq-n: R(m, n)=S(m, n)
$$

We specialize (25) to $m=-n-1$. Since

$$
\begin{aligned}
S(-n-1, n)= & -(n+1) n \varphi_{1}+(n+1) \varphi_{-n, n} \varphi_{1}+\sum_{j=2}^{n} \frac{n+1}{j} \varphi_{-(n+1)+j, n} \varphi_{j} \\
& +\sum_{j=1}^{n-1} \frac{n}{j} \varphi_{-(n+1), n-j} \varphi_{j}
\end{aligned}
$$

and $\varphi_{1}=\Theta(z)$, the Eq. (25) yields

$$
\varphi_{-n, n}-n=\frac{1}{(n+1) \Theta}\left(R(-n-1, n)-\sum_{j=2}^{n} \frac{n+1}{j} \varphi_{-(n+1)+j, n} \varphi_{j}-\sum_{j=1}^{n-1} \frac{n}{j} \varphi_{-(n+1), n-j} \varphi_{j}\right)
$$

The term in the bracket on the right lies in $\mathrm{QJac}_{-1, n+1 / 2}$ by inspection. Moreover, again by inspection it vanishes at $z=0$. Hence it must be divisible in algebra of quasi-Jacobi forms by $\Theta(z)$. This gives $\varphi_{-n, n}-n \in \mathrm{QJac}_{0, n}$.

Remark 4 The proof yields more information. For $m \neq-n$ we have $\frac{d}{d A} \varphi_{m, n}=$ $R(m, n)$ by (19). Using that $R(m, n)=S(m, n)$ for all $m \neq-n$ we find the anomaly equation

$$
\frac{d}{d A} \varphi_{-m, n}=\frac{-m \cdot n}{-m+n} \varphi_{-m+n}+\sum_{j=1}^{m-1} \frac{m}{j} \varphi_{-m+j, n} \varphi_{j}+\sum_{j=1}^{n-1} \frac{n}{j} \varphi_{-m, n-j} \varphi_{j}
$$


where $m, n>0$ and $m \neq-n . \triangle$

\subsection{Holomorphic anomaly equations II}

We finally derive the precise modular properties of the functions $\varphi_{m, n}$ in terms of holomorphic anomaly equations.

Proposition 4.5 For all $m, n \in \mathbb{Z}$ we have

(a) $\frac{d}{d G_{2}} \varphi_{m, n}=2 \varphi_{m} \varphi_{n}$.

(b) $\frac{d}{d A} \varphi_{m, n}=\frac{m \cdot n}{m+n} \varphi_{m+n}+\sum_{i+j=m} \frac{|m|}{j} \varphi_{i, n} \varphi_{j}+\sum_{i+j=n} \frac{|n|}{j} \varphi_{m, i} \varphi_{j}$

with the convention in $(b)$ that the first term vanishes if $m+n=0$ and that in a sum with condition $i+j=\ell$ (for $\ell=m$ or $\ell=n$ ) we sum over all positive $i, j$ if $\ell$ is positive, and over all negative $i, j$ if $\ell$ is negative.

Proof Part (a) follows from the defining differential equation (5) by applying $d / d G_{2}$. In part (b) by Proposition 4.4 and Remark 4 we only need to prove the case $m=-n$. For that we restrict ourself to the region $m<0$ and $n>0$. Applying $d / d A$ to (5) yields

$$
D_{z} \varphi_{m, n}+D_{\tau} \frac{d}{d A} \varphi_{m, n}=\frac{d}{d A}\left(m n \varphi_{m} \varphi_{n} F+\left(D_{\tau} \varphi_{m}\right)\left(D_{\tau} \varphi_{n}\right)\right)
$$

The right-hand side and the first term on the left-hand side are polynomial in $m$ and $n$ (in the considered region). Hence $\frac{d}{d A} \varphi_{m, n}$ is polynomial in $m, n$ up to a constant in $q .^{7}$ Let $T(u, v)$ be the polynomial series such that

$$
T(m, n)=\frac{m \cdot n}{m+n} \varphi_{m+n}+\sum_{i+j=m} \frac{|m|}{j} \varphi_{i, n} \varphi_{j}+\sum_{i+j=n} \frac{|n|}{j} \varphi_{m, i} \varphi_{j}
$$

for all $m \neq-n$ in the region. We already know $T(m, n)=\frac{d}{d A} \varphi_{m, n}$ for all $m \neq-n$ so by the polynomiality of $\frac{d}{d A} \varphi_{m, n}$ we get for all $m, n$ in the region

$$
T(m, n)=\frac{d}{d A} \varphi_{m, n}+c_{m, n}(z)
$$

for some $c_{m, n}(z)$ which does not depend on $q$. Specializing to $m=-n$ we see

$$
\frac{d}{d A} \varphi_{-n, n}+c_{-n, n}(z)=T(-n, n)=-n^{2} z+\sum_{i+j=-n} \frac{n}{j} \varphi_{i, n} \varphi_{j}+\sum_{i+j=n} \frac{n}{j} \varphi_{-n, i} \varphi_{j} .
$$

\footnotetext{
7 There is a small subtlety here since at first it only follows that $\frac{d}{d A} \varphi_{m, n}$ is a power series in $z, q$ whose coefficients are polynomial in $m, n$. But then $\frac{d}{d A} \varphi_{m, n}$ is a quasi-Jacobi form for every $m, n$ so that this actually has to be a power series in $z$ with coefficients which are polynomials with coefficients quasi-modular forms (of determined weight).
} 
But $\frac{d}{d A} \varphi_{-n, n}$ is homogeneous as a quasi-Jacobi form of weight -1 and index $n$. Hence the constant terms in $q$ on both sides must match up and so as desired

$$
\frac{d}{d A} \varphi_{-n, n}=\sum_{i+j=-n} \frac{n}{j} \varphi_{i, n} \varphi_{j}+\sum_{i+j=n} \frac{n}{j} \varphi_{-n, i} \varphi_{j}
$$

Remark 5 Once we know that $\varphi_{n,-n}$ is quasi-Jacobi and know its $A$-derivative it is not difficult to derive a recursive formula for it (ignoring that we already obtained a formula in the proof of Theorem 1.3). Indeed, consider the defining differential equation

$$
D_{\tau} \varphi_{m, n}=m n \varphi_{m} \varphi_{n} F+\left(D_{\tau} \varphi_{m}\right)\left(D_{\tau} \varphi_{n}\right)
$$

Applying $\frac{d}{d A}$ twice and using the commutation relations we get

$$
(|m|+|n|) \varphi_{m, n}+2 D_{z} \frac{d}{d A} \varphi_{m, n}+D_{\tau}\left(\frac{d}{d A}\right)^{2} \varphi_{m, n}=\left(\frac{d}{d A}\right)^{2}\left(m n \varphi_{m} \varphi_{n} F+\left(D_{\tau} \varphi_{m}\right)\left(D_{\tau} \varphi_{n}\right)\right)
$$

Since $\left(\frac{d}{d A}\right)^{i} \varphi_{m, n}$ is determined recursively from functions indexed by $m^{\prime}, n^{\prime}$ with $m^{\prime}+$ $n^{\prime}<m+n$ this yields one more formula for $\varphi_{m, n} \cdot \triangle$

\section{The classical Kaneko-Zagier equation}

The differential equation introduced by Kaneko and Zagier [9] can be characterized among quadratic differential equations as those for which the solution space is invariant under the modular transformation for the full modular group, so that it is essentially unique [7]. If one however considers congruence subgroups, further differential equations of the same type have been found by Kaneko and Koike [8]. In this section we give a general construction which takes as input a meromorphic Jacobi form of weight -1 and gives as output a differential equation of Kaneko-Zagier type. The two Kaneko-Zagier equations above and our case studied in this paper are all given by this construction. $^{8}$

\subsection{A general construction}

A general recipe to construct Kaneko-Zagier type differential equations is as follows. Let $g$ be a meromorphic Jacobi form of weight -1 . Define

$$
E(\tau)=\frac{D_{\tau} g(\tau)}{g(\tau)} \text { and } H(\tau)=\frac{D_{\tau}^{2} g(\tau)}{g(\tau)} .
$$

\footnotetext{
8 A certain differential equation for index 1 Jacobi forms was studied by Kiyuna [10] and was called a Kaneko-Zagier type equation. Howver, since it is of 4-th order it does not fit our framework.
} 
By construction $E$ and $H$ are meromorphic quasi-Jacobi forms. For all $m \geq 1$ we consider the differential equation

$$
D_{\tau}^{2} g_{m}=m^{2} H(\tau) g_{m}
$$

To obtain the connection to the classical presentation, we set $m=k+1$, and consider

$$
f_{k}=g_{k+1} / g^{k+1}
$$

which is of weight $k$. The corresponding differential equation for $f_{k}$ reads

$$
D_{\tau}^{2} f_{k}+2(k+1) E(\tau) D_{\tau} f_{k}+k(k+1)\left(E(\tau)^{2}-H(\tau)\right) f_{k}=0 .
$$

For this choice of $g$ (and hence of $E$ ), we define a modified Serre derivative

$$
\theta_{g}=D_{\tau}+E \mathrm{wt}
$$

The operator $\theta_{g}$ is a derivation vanishing on $g$. Moreover, the above differential equation can be rewritten as

$$
\theta_{g}^{2} f_{k}=H \mathrm{wt}(\mathrm{wt}+2) f_{k}
$$

We give several examples:

(0) In this paper we considered the case $g(z, \tau)=\Theta(z, \tau)$ (which contains the cases $g(\tau)=\Theta(a, \tau)$ for any $a \in \mathbb{Q})$.

(1) For the classical Kaneko-Zagier equation we let

$$
g(\tau)=\frac{1}{\eta(\tau)^{2}}
$$

and get $H(\tau)=E_{4}(\tau) / 144$. The operator $\theta_{g}$ is the Serre derivative.

(2) For the differential equation studied in [8] we take

$$
g(\tau)=\frac{1}{\eta(\tau) \eta(2 \tau)}
$$

and get

$$
E(\tau)=\frac{1}{24}\left(E_{2}(\tau)+2 E_{2}(2 \tau)\right) \quad 2^{6} H(\tau)=\frac{1}{5}\left(E_{4}(\tau)+4 E_{4}(2 \tau)\right) .
$$

The operator $\theta_{g}$ matches the derivative operator of [8, Sec. 2]. Unpublished work by Tomoaki Nakaya [13, Section 3.5] shows that in this case

$$
f_{k}=\operatorname{Res}_{z=0}\left(D_{z} \frac{\Theta(2 z, 2 \tau)}{\Theta(z, \tau)^{2}}\right)^{-(k+1) / 2}
$$


is a solution of the differential equation (26) for all $k \geq 1$.

\subsection{Recursive construction of the solutions}

Let $f_{k}$ and $f_{l}$ be two solutions of (26) of weight $k$ and $l$ respectively. We write

$$
\begin{aligned}
{[f, h] } & :=k \theta_{g}(f) h-l f \theta_{g}(h) \\
& =k D_{\tau}(f) h-l f D_{\tau}(h)
\end{aligned}
$$

which specializes to the first Rankin-Cohen bracket on modular forms.

Proposition 5.1 We have

$$
\begin{aligned}
\theta_{g}\left[f_{k}, f_{l}\right] & =\frac{k-l}{l+2}\left[f_{k}, \theta_{g}\left(f_{l}\right)\right] \\
\theta_{g}^{2}\left[f_{k}, f_{l}\right] & =(k-l)(k-l-2) H\left[f_{k}, f_{l}\right]+k(k-l) f_{k}\left[f_{l}, H\right] .
\end{aligned}
$$

Proof This follows from a direct computation.

Corollary 5.2 Suppose that $\left[f_{l}, H\right]=0$. Then

$$
\left[f_{k}, f_{l}\right] g^{2 l+4} \text { and }\left[f_{k} g^{2 k+2}, f_{l}\right] g^{-2 k-2}
$$

are solutions of (26) of weight $k-l-2$ and $k+l+2$ respectively.

Hence if a function $f_{l}$ as in the corollary exists, then from any given solution we can recursively write down solutions of (26) with weight in the same residue class modulo $l$.

Example 5.3 For the classical Kaneko-Zagier equation we can take $f_{l}=E_{4}$. Then indeed $\left[f_{l}, H\right]=0$, so that if $f_{k}$ is a solution we have that $\left[f_{k}, E_{4}\right] / \Delta$ is a solution of weight $k-6$, and $\left[f_{k} \eta^{-4 k-4}, E_{4}\right] \eta^{4 k+4}$ is a solution of weight $k+6$. The first of these equations can also be found in [7, Proposition 1(i)].

Example 5.4 For the Kaneko-Zagier equation in Example (2) we can take $f_{l}=$ $2 E_{2}(2 \tau)-E_{2}(\tau)$. Then, indeed $\left[f_{l}, H\right]=0$, so solutions can be constructed 4periodically, compare also with [8].

Remark 6 However, in the differential equation of Example (0) considered in this paper, it turns out that the recursive structure described in Corollary 5.2 does not exist. To see this, suppose (for our general family of Kaneko-Zagier equations) that there exists a solution $f_{l}$ and that moreover we have $\left[f_{l}, H\right]=0$. Then the condition $\left[f_{l}, H\right]=0$ is equivalent to

$$
\theta_{g} f_{l}=\frac{l}{4}\left(\frac{D_{\tau}^{3} g}{D_{\tau}^{2} g}+3 \frac{D_{\tau} g}{g}\right) f_{l} .
$$


Applying $\theta_{g}$ to this equation and using the differential equation for the left hand side, we obtain

$$
\begin{aligned}
16(l+2) \frac{D_{\tau}^{2} g}{g}= & 4 \frac{D_{\tau}^{4} g}{D_{\tau}^{2} g}+12 \frac{D_{\tau}^{2} g}{g}+(l-4)\left(\frac{D_{\tau}^{3} g}{D_{\tau}^{2} g}\right)^{2}+2(3 l+4) \frac{D_{\tau}^{3} g}{D_{\tau}^{2} g} \frac{D_{\tau} g}{g} \\
& +3(3 l+4)\left(\frac{D_{\tau} g}{g}\right)^{2} .
\end{aligned}
$$

For $g=\eta^{-2}$ this equation is only satisfied if $l=4$, and for $g=(\eta(\tau) \eta(2 \tau))^{-1}$ only if $l=2$. However, for $g=\Theta(z)$ this equation is never satisfied, so Corollary 5.2 cannot be applied. $\triangle$

Acknowledgements We thank A. Oblomkov for pointing out the polynomial dependence in the $\varphi_{m}$, and R. Pandharipande for the many things we learned from him about the Gromov-Witten theory of K3 surfaces. The second author was partially funded by the DFG Grant OB 512/1-1. The third author was partially supported by NSF Grant 1807079.

Funding Open Access funding enabled and organized by Projekt DEAL.

Open Access This article is licensed under a Creative Commons Attribution 4.0 International License, which permits use, sharing, adaptation, distribution and reproduction in any medium or format, as long as you give appropriate credit to the original author(s) and the source, provide a link to the Creative Commons licence, and indicate if changes were made. The images or other third party material in this article are included in the article's Creative Commons licence, unless indicated otherwise in a credit line to the material. If material is not included in the article's Creative Commons licence and your intended use is not permitted by statutory regulation or exceeds the permitted use, you will need to obtain permission directly from the copyright holder. To view a copy of this licence, visit http://creativecommons.org/licenses/by/4.0/.

\section{References}

1. Bryan, J., Oberdieck, G.: CHL Calabi-Yau threefolds: curve counting, Mathieu moonshine and Siegel modular forms. Commun. Number Theory Phys. 14(4), 785-862 (2020)

2. Beauville, A.: Counting rational curves on $K 3$ surfaces. Duke Math. J. 97(1), 99-108 (1999)

3. Bryan, J., Leung, N.C.: The enumerative geometry of $K 3$ surfaces and modular forms. J. Am. Math. Soc. 13(2), 371-410 (2000)

4. Eichler, M., Zagier, D.: The Theory of Jacobi Forms, Progress in Mathematics, vol. 55. Birkhäuser Boston Inc., Boston (1985)

5. Gritsenko, V., Skoruppa, N., Zagier, D.: Theta Blocks. arXiv:1907.00188

6. Janda, F., Pandharipande, R., Pixton, A., Zvonkine, D.: Double ramification cycles on the moduli spaces of curves. Publ. Math. Inst. Hautes Études Sci. 125, 221-266 (2017)

7. Kaneko, M., Koike, M.: On modular forms arising from a differential equation of hypergeometric type, Rankin memorial issues. Ramanujan J. 7(1-3), 145-164 (2003)

8. Kaneko, M., Koike, M.: Quasimodular solutions of a differential equation of hypergeometric type. In: Hashimoto, K., Miyake, K., Nakamura, H. (eds.) Galois Theory and Modular Forms, Developments in Mathematics, vol. 11, pp. 329-336. Kluwer Academic Publishers, Boston (2004)

9. Kaneko, M., Zagier, D.: Supersingular $j$-invariants, hypergeometric series, and Atkin's orthogonal polynomials. Computational perspectives on number theory (Chicago, IL, 1995), AMS/IP Studies in Advanced Mathematics, vol. 7, pp. 97-126. American Mathematical Society, Providence (1998)

10. Kiyuna, T.: Kaneko-Zagier type equation for Jacobi forms of index 1. Ramanujan J. 39(2), 347-362 (2016) 
11. Libgober, A.: Elliptic genera, real algebraic varieties and quasi-Jacobi forms, Topology of Stratified Spaces, Mathematical Sciences Research Institute Publications, vol. 58, pp. 95-120. Cambridge University Press, Cambridge (2011)

12. Maulik, D., Pandharipande, R., Thomas, R.P.: Curves on $K 3$ surfaces and modular forms, with an appendix by A. Pixton. J. Topol. 3(4), 937-996 (2010)

13. Nakaya, T.: On classical elliptic functions and automorphic differential equations. Unpublished conference proceedings of the 13th Fukuoka mathematical research conference, communicated by Masanobu Kaneko

14. Oberdieck, G., Pixton, A.: Gromov-Witten theory of elliptic fibrations: Jacobi forms and holomorphic anomaly equations. Geom. Topol. 23(3), 1415-1489 (2019)

15. Oberdieck, G.: Gromov-Witten invariants of the Hilbert scheme of points of a K3 surface. Geom. Topol. 22(1), 323-437 (2018)

16. Oberdieck, G., Pandharipande, R.: Curve counting on $K 3 \times E$, the Igusa cusp form $\chi_{10}$, and descendent integration. In: Faber, C., Farkas, G., van der Geer, G. (eds.) K3 Surfaces and Their Moduli, Birkhauser Progress in Mathematics, vol. 315, pp. 245-278. Birkhauser, Basel (2016)

17. Oberdieck, G., Pixton, A.: Holomorphic anomaly equations and the Igusa cusp form conjecture. Invent. Math. 213(2), 507-587 (2018)

18. Oberdieck, G.: Gromov-Witten theory of $\mathrm{K} 3 \times \mathbb{P}^{1}$ and quasi-Jacobi forms. Int. Math. Res. Not. IMRN 16, 4966-5011 (2019)

19. Weil, A.: Elliptic functions according to Eisenstein and Kronecker, Ergebnisse der Mathematik und ihrer Grenzgebiete, Band 88. Springer, Berlin-New York, ii+93 pp (1976)

Publisher's Note Springer Nature remains neutral with regard to jurisdictional claims in published maps and institutional affiliations. 